Journal of Agricultural Sciences
(Tarim Bilimleri Dergisi)

\title{
An efficient Regeneration Protocol for in vitro Direct Organogenesis in Einkorn (Triticum monococcum L.) Wheat
}

\author{
Günce ŞAHIN ${ }^{\mathrm{a} *}$ (iD), Mehmet ÖRGEÇ ${ }^{\mathrm{iD}}$, Nusret ZENCIRCI ${ }^{\mathrm{a}}$ (D) \\ ${ }^{a}$ Bolu Abant Izzet Baysal University, Faculty of Arts and Science, Department of Biology, Bolu, TURKEY \\ ARTICLE INFO \\ Research Article \\ Corresponding Author: Günce ŞAHIN, E-mail: guncesahin@gmail.com \\ Received: 5 March 2021 / Revised: 17 July 2021 / Accepted: 14 July 2021 / Online: 01 September 2022 \\ Cite this article \\ SAHIN G, ÖRGEÇ M, ZENCIRCI N (2022). An efficient Regeneration Protocol for in vitro Direct Organogenesis in Einkorn (Triticum monococcum L.) Wheat. Journal of Agricultural \\ Sciences (Tarim Bilimleri Dergisi), 28(3):412-422. DOI: 10.15832/ankutbd.891812
}

\section{ABSTRACT}

Coleoptile, leaf, and root explants of the einkorn (Triticum monococcum ssp. monococcum) were cultured in vitro to obtain an efficient plant regeneration protocol through direct shoot formation by using different combinations and concentrations of various plant growth regulators. A total of 180 different auxin and cytokinin combinations were tested for regeneration. Shoot formation was not observed with the root and leaf explants. Shoot formation was obtained only from the coleoptile explants, with a mean of $1.20 \pm 0.24$ shoots/explant and $86.60 \%$ of shoot formation frequency and with a $1.20 \pm 0.53$ shoots/explant and $80.00 \%$ shoot formation frequency on medium supplemented with $0.5 \mathrm{mg} \mathrm{L}^{-1} \mathrm{TDZ}$ and $1 \mathrm{mg} \mathrm{L}^{-1} \mathrm{TDZ}$ plus $1 \mathrm{mg} \mathrm{L}^{-1} \mathrm{NAA}$, respectively. The shoots were subcultured on the MS medium containing the most effective hormonal combination concurrently continued to shoot and root formation for 45 days. It is noteworthy that $3.66 \pm 0.66$ shoots per explant were induced by MS, which contained $1 \mathrm{mg} \mathrm{L}^{-1} \mathrm{TDZ}$ plus $1 \mathrm{mg} \mathrm{L}^{-1} \mathrm{NAA}$ and $2.0 \mathrm{mg} \mathrm{L}^{-1}$ KIN plus $0.5 \mathrm{mg} \mathrm{L}^{-1} \mathrm{NAA}$ for 45 days. Of the different auxin concentrations tested for rooting, $2.0 \mathrm{mg} \mathrm{L}^{-1}$ IAA was predominant, with the greatest number of roots $(12.33 \pm 0.88)$ produced per regenerated shoot. Finally, these well-developed plantlets were acclimatized with a $100 \%$ success rate and were transferred to the ex vitro conditions. A highly efficient regeneration protocol for einkorn wheat was developed using somatic tissue as an explant source for the first time.

Keywords: Coleoptile, Direct regeneration protocol, Tissue culture, Einkorn wheat (Triticum monococcum ssp. monococcum)

\section{Introduction}

Micropropagation is used commercially worldwide, but the capacity of plant regeneration and somatic organogenesis varies greatly among species (Bidabadi \& Jain 2020). Wheat includes more than 20 cultivated species (Goncharov 2011), however, in vitro plant regeneration ability of many wheat species has not been studied until recently. Triticum monococcum ssp. monococcum (einkorn) wheat, diploid ancestral wheat, intervened in the spread and rise of agriculture for several thousand years until more productive polyploid wheat was replaced with it (Nesbitt \& Samuel 1996). But nowadays, the renewed interest in studies related to this cereal is on the rise because of its putative low allergenicity, disease resistance properties, and lower gluten but higher lutein and protein content (Hidalgoa et al. 2006; Özgen et al. 2017). Moreover, it has been recently included in modern wheat breeding programs, as donors of stress resistance genes (Nevo 2011; Login \& Reif 2014; Alikina et al. 2016).

Improving crops to create genetic variability and increase the number of desirable germplasms is dependent on the establishment of a highly regenerative tissue culture system for many plant species, particularly cereals. Many factors can affect this system, such as culture medium, growth conditions, genotypes, and explant types. Currently, in tissue culture studies on wheat and other cereal crops, immature-mature embryos and inflorescences have been traditionally used as the most suitable explant source and the regeneration capacity of plants has been reported with varying degrees of success (Benlioğlu \& Birsin 2017).

In comparison to other wheat tissues, immature zygotic embryos are the most commonly and efficiently used explants for plant regeneration in hexaploid bread wheat, tetraploid durum wheat, and only a few numbers of studies that have been conducted on diploid wheats (Miroshnichenko et al. 2017). The standard technique entails the cultivation of immature tissues on 2,4-Dcontaining media in the dark, followed by plant differentiation in the light on media devoid of phytohormones (Fennell et al. 1996; Tama's et al. 2004; Chauhan et al. 2007; Miroshnichenko et al. 2016). However, this conventional protocol is often ineffective for many wheat genotypes due to the inability to regenerate entire plants on a regular basis. Moreover, the cultivation of donor plants to obtain embryos involves the expenditure of much time and money. 
Although using somatic tissues as an explant source makes it possible to obtain a great amount of material regardless of these short-comings, ventures to determine a reliable plant regeneration protocol using somatic cell cultures for diploid wheat species did not produce positive results (Alikina et al. 2016). Similarly, no shoot differentiation was observed in somatic cell cultures of tetraploid and hexaploid wheat genotypes (Lazar et al. 1983; Bi \& Wang 2008; Özgen et al. 2017). Therefore, even after many years of research, especially in the genotypes of wheat, screening of germplasms in vitro response is very important for biotechnological applications.

The objective of this study was to investigate a highly effective in vitro regeneration protocol for the einkorn wheat via adventitious shoot formation from the root, coleoptile, and leaf explants cultured on the MS medium containing different combinations and concentrations of plant growth regulators for the first time. Direct shoot formation from somatic tissue is a remarkable feature of this regeneration protocol.

\section{Material and Methods}

\subsection{Plant materials and growth condition}

Seeds of the einkorn (Triticum monococcum ssp. monococcum) wheat were collected from İhsangazi / Kastamonu, Turkey in 2014-2015. Seeds were disinfected with $100 \mathrm{ml}$ distilled water containing 5 drops of Tween20 (Merck, Darmstadt, Germany) for $1 \mathrm{~min}$, then sterilized with $40 \%$ commercial bleach ( $4.6 \% \mathrm{NaClO}$; Domestos, Istanbul, Turkey) for 15 min, and finally washed three times with sterile $\mathrm{dH}_{2} \mathrm{O}$ (Örgeç et al. 2018). Seeds (20 seeds / $100 \mathrm{~mm} \times 15 \mathrm{~mm}$ Petri dish) were cultured on the MS (Duchefa-Haarlem, Netherlands) medium (pH: 5.8), (Murashige \& Skoog 1962) containing 2.5\% (w/v) sucrose (Merck Darmstadt, Germany) and $0.75 \%$ (w/v) agar (Duchefa-Haarlem, Netherlands). The seeds were grown for germination under a $16 / 8 \mathrm{~h}$ photoperiod at $24 \pm 2{ }^{\circ} \mathrm{C}$ (climate room conditions) for 10 days.

\subsection{Shoot regeneration}

Five to eight mm segments of the leaf, coleoptile, and root sliced from ten-days old germinated seedlings were cultured on the MS medium containing different concentrations (from 0.5 to $3 \mathrm{mg} \mathrm{L}^{-1}$ ) of IAA (indole acetic acid) (Duchefa-Haarlem), NAA ( $\alpha$-naphthalene acetic acid) or 2.4-D (2.4-dichloro phenoxy acetic acid) (Sigma-Aldrich, Steinhem, Germany) and combined with TDZ (thidiazuron) (Duchefa-Haarlem), KIN (kinetin) or BAP (6-benzyl amino purine) ranging from 0.5 to $3 \mathrm{mg} \mathrm{L}^{-1}$. The mean number of shoots/explant and the percentage (\%) of developing shoots were enrolled 15 days after the culture grown under climate room conditions ( $16 \mathrm{~h}$ light: $8 \mathrm{~h}$ dark photoperiod at $23 \pm 2{ }^{\circ} \mathrm{C}$ ), respectively. After a period of 15 days, plantlets were transferred to MS which contained the best response hormone combination to obtain more shoots and observed for up to 45 days. The data were recorded continuously.

\subsection{Rooting of the shoots, hardening, and acclimatization}

Forty-five days old shoots were transferred to MS medium supplemented with IAA (ranging from 0.5 to $5 \mathrm{mg} \mathrm{L}^{-1}$ ) to observe root formation for 30 days. After 30 days, the mean numbers of roots/explant were recorded. Then, well-developed plantlets were transferred to Magenta vessels $(77 \mathrm{~mm} \times 77 \mathrm{~mm} \times 97 \mathrm{~mm})$ containing a mixture of vermiculite and soil (1:2) for acclimatization. The plantlets were kept in the climate room for 1 week. After 1 week, the plantlets were transferred to room conditions.

\subsection{Statistical analysis}

The data were statistically analyzed using SPSS 16.0 (SPSS Inc., Chicago, IL, USA). Differences in means \pm SD (standard deviation) were analyzed using Duncan's multiple range test at $\mathrm{P}<0.05$.

\section{Results and Discussion}

This study provided an efficient protocol for the direct formation of shoots from different explants, the first as such report for einkorn wheat. The traditional method for wheat propagation is using an immature and mature embryo as an explant source. Several studies have been conducted to determine the indirect regeneration protocol from immature and mature embryo cultures in the einkorn wheat (Yang et al. 2015; Alikina et al. 2016; Miroshnichenko et al. 2016). However, there are no reports on einkorn cultures in which improvement in direct shoot production using somatic tissues has been achieved. For this reason, two sets of experiments were carried out. In the first set, the regeneration capacity of three different explant sources (leaf, coleoptile, and root) cultured on the MS medium containing various concentrations of BAP, KIN, or TDZ, in combination with NAA, 2.4$\mathrm{D}$, or IAA ranging from 0.5 to $3 \mathrm{mg} \mathrm{L}^{-1}$ were obtained. In the second set, the most effective hormone combinations were selected to increase the number of shoots.

A total of 180 different hormone combinations were tested for regeneration and variable growth rates were recorded. Control treatments without PGRs produced no shoots. In all tested hormone combinations, shoot formation was not observed with leaf 
and root explants. It was observed that coleoptile was the best as an explant source referring to both the percentages of explants forming shoots and the mean number of shoots/explant with TDZ (Table 1 and Figure 1). The TDZ/NAA combination was more effective than the TDZ/IAA or TDZ/2.4-D combination for shoot propagation in this study. When explants were cultured on MS containing KIN or BA in combination with NAA or 2.4-D, the frequency of formation and the number of shoots were found at approximately the same levels. Optimum shoot formation was detected from coleoptile explants, with a mean of $1.20 \pm 0.24$ shoots/explant and $86.60 \%$ shoot formation percentage and with a $1.20 \pm 0.53$ shoots/explant and $80 \%$ shoot formation percentage on medium supplemented with $0.5 \mathrm{mg} \mathrm{L}^{-1} \mathrm{TDZ}$ and $1 \mathrm{mg} \mathrm{L}^{-1} \mathrm{TDZ}+1 \mathrm{mg} \mathrm{L}^{-1} \mathrm{NAA}$, respectively (Table 1).

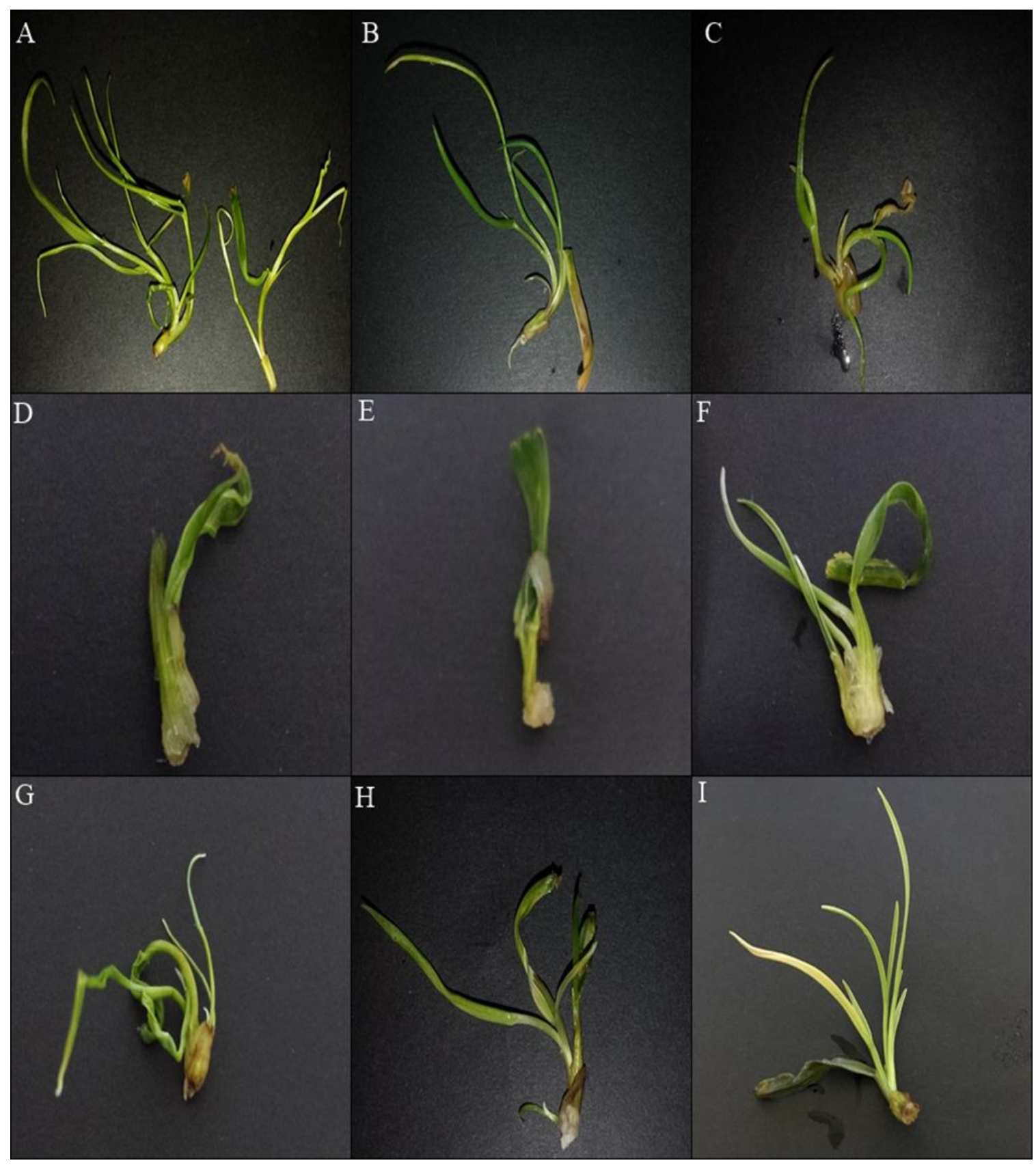

Figure 1- The effects of various concentrations of BA, KIN or TDZ, in combination with NAA, 2,4-D or IAA ranging from 0.5 to $3 \mathrm{mg} \mathrm{L}^{-1}$ on shoot formation (A) $0.5 \mathrm{mg} \mathrm{L}^{-1} \mathrm{TDZ}$. (B) $1 \mathrm{mg} \mathrm{L}^{-1} \mathrm{KIN}$. (C) $2 \mathrm{mg} \mathrm{L}^{-1} \mathrm{BA}+0.5 \mathrm{mg} \mathrm{L}^{-1}$ IAA. (D) $2 \mathrm{mg} \mathrm{L}^{-1} \mathrm{TDZ}^{-1}$ mg L ${ }^{-1}$ 2.4-D. (E) $0.5 \mathrm{mg} \mathrm{L}^{-1} \mathrm{KIN}+1 \mathrm{mg} \mathrm{L}^{-1} 2.4-D$ (F) $3 \mathrm{mg} \mathrm{L}^{-1} \mathrm{BA}+2 \mathrm{mg} \mathrm{L}^{-1}$ 2.4-D. (G) $1 \mathrm{mg} / 1 \mathrm{TDZ}+1 \mathrm{mg} / \mathrm{l} \mathrm{NAA}$ (H) 2.0 mg/l KIN+0.5 mg/l NAA. (I) $0.5 \mathrm{mg} / \mathrm{l} \mathrm{BA}+2.0 \mathrm{mg} \mathrm{NAA}$ 
Table 1- Shoot regeneration from coleoptile explants cultured on MS medium containing different combinations of KIN, TDZ or BA with NAA, 2.4 D or IAA. Mean-values with the same letters within vertical columns are not significantly different $(\mathbf{P}>0.05)$. Control means are with no PGR treatment

\begin{tabular}{|c|c|c|c|c|c|c|c|c|}
\hline $\begin{array}{c}\text { PGRs } \\
\left(m g L^{-1)}\right.\end{array}$ & $\begin{array}{c}\text { Mean number } \\
\text { of } \\
\text { shoots/explant }\end{array}$ & $\begin{array}{c}\text { Shoot } \\
\text { formation } \\
\text { percentage }(\%)\end{array}$ & $\begin{array}{c}\text { PGRs } \\
\left(m g L^{-1)}\right.\end{array}$ & $\begin{array}{c}\text { Mean number } \\
\text { of } \\
\text { shoots/explant }\end{array}$ & $\begin{array}{c}\text { Shoot } \\
\text { formation } f \\
\text { percentage }(\%)\end{array}$ & $\begin{array}{c}\text { PGRs } \\
\left(m g L^{-1)}\right.\end{array}$ & $\begin{array}{c}\text { Mean number } \\
\text { of } \\
\text { shoots/explant }\end{array}$ & $\begin{array}{l}\text { Shoot formation } \\
\text { percentage (\%) }\end{array}$ \\
\hline Control & - & - & & - & - & & - & - \\
\hline $\begin{array}{c}T D Z- \\
I A A\end{array}$ & & & $\begin{array}{c}\text { KIN - } \\
I A A\end{array}$ & & & $\begin{array}{c}B A- \\
I A A\end{array}$ & & \\
\hline $0.5-0$ & $1.20 \pm 0.24^{\mathrm{a}}$ & 86.60 & $0.5-0$ & $0.89 \pm 0.38^{\mathrm{abcde}}$ & 86.60 & $0.5-0$ & $0.64 \pm 0.24^{\text {bcdefght }}$ & 46.60 \\
\hline $1.0-0$ & $0.78 \pm 0.23^{\mathrm{abcdefg}}$ & 60.00 & $1.0-0$ & $0.93 \pm 0.34^{\mathrm{abcd}}$ & 73.30 & $1.0-0$ & $0.64 \pm 0.29^{\text {bcdefgh } 1}$ & 40.00 \\
\hline $2.0-0$ & $0.93 \pm 0.38^{\mathrm{abcd}}$ & 66.60 & $2.0-0$ & $0.87 \pm 0.30^{\text {abcde }}$ & 73.30 & $2.0-0$ & $0.78 \pm 0.21^{\text {abcdefg }}$ & 53.30 \\
\hline $3.0-0$ & $0.82 \pm 0.27^{\text {abcdef }}$ & 73.30 & $3.0-0$ & $0.71 \pm 0.22^{\text {bcdefgh }}$ & 66.60 & $3.0-0$ & $0.62 \pm 0.25^{\text {bcdefgh } 1}$ & 46.60 \\
\hline $0.5-0.5$ & $0.73 \pm 0.23^{\mathrm{abcdefg}}$ & 60.00 & $0.5-0.5$ & $0.40 \pm 0.22^{\text {efght }}$ & 40.00 & $0.5-0.5$ & $0.73 \pm 0.11^{\text {abcdefg }}$ & 66.60 \\
\hline $1.0-0.5$ & $0.80 \pm 0.20^{\text {abcdef }}$ & 66.60 & $1.0-0.5$ & $0.87 \pm 0.30^{\text {abcde }}$ & 60.00 & $1.0-0.5$ & $0.67 \pm 0.50^{\text {bcdefgh }}$ & 53.30 \\
\hline $2.0-0.5$ & $0.73 \pm 0.61^{\text {abcdefg }}$ & 53.30 & $2.0-0.5$ & $0.33 \pm 0.11^{\text {fght }}$ & 33.30 & $2.0-0.5$ & $0.87 \pm 0.30^{\text {abcde }}$ & 60.00 \\
\hline $3.0-0.5$ & $0.73 \pm 0.12^{\text {bcdefg }}$ & 60.00 & $3.0-0.5$ & $0.40 \pm 0.22^{\text {efght }}$ & 33.30 & $3.0-0.5$ & $1.00 \pm 0.40^{\mathrm{abc}}$ & 60.00 \\
\hline $0.5-1.0$ & $0.60 \pm 0.20^{\text {bcdefght }}$ & 60.00 & $0.5-1.0$ & $0.53 \pm 0.11^{\text {cdefght }}$ & 46.60 & $0.5-1.0$ & $0.53 \pm 0.11^{\text {cdefghı }}$ & 53.30 \\
\hline $1.0-1.0$ & $0.73 \pm 0.12^{\text {abcdefg }}$ & 60.00 & $1.0-1.0$ & $0.47 \pm 0.46^{\text {defght }}$ & 20.00 & $1.0-1.0$ & $0.47 \pm 0.11^{\text {defgh } 1}$ & 46.60 \\
\hline $2.0-1.0$ & $0.60 \pm 0.20^{\text {bcdefght }}$ & 60.00 & $2.0-1.0$ & $0.73 \pm 0.11^{\text {abcdefg }}$ & 33.30 & $2.0-1.0$ & $0.33 \pm 0.11^{\text {fght }}$ & 33.30 \\
\hline $3.0-1.0$ & $0.60 \pm 0.20^{\text {bcdefght }}$ & 60.00 & $3.0-1.0$ & $0.20 \pm 0.11^{\mathrm{h}}$ & 20.00 & $3.0-1.0$ & $0.47 \pm 0.30^{\text {defghı }}$ & 40.00 \\
\hline $0.5-2.0$ & $0.73 \pm 0.12^{\text {abcdefg }}$ & 60.00 & $0.5-2.0$ & $0.33 \pm 0.23^{\text {fght }}$ & 26.60 & $0.5-2.0$ & $0.67 \pm 0.23^{\text {bcdefgh }}$ & 60.00 \\
\hline $1.0-2.0$ & $0.87 \pm 0.50^{\text {abcde }}$ & 53.30 & $1.0-2.0$ & $0.33 \pm 0.30^{\text {fght }}$ & 33.30 & $1.0-2.0$ & $0.20 \pm 0.11^{\mathrm{h} 1}$ & 20.00 \\
\hline $2.0-2.0$ & $0.73 \pm 0.23^{\text {abcdefg }}$ & 73.30 & $2.0-2.0$ & $0.40 \pm 0.22^{\text {efght }}$ & 26.60 & $2.0-2.0$ & $0.53 \pm 0.11^{\text {cdefght }}$ & 46.60 \\
\hline $3.0-2.0$ & $0.67 \pm 0.12^{\text {bcdefgh }}$ & 73.30 & $3.0-2.0$ & $0.27 \pm 0.23^{\text {ght }}$ & 26.60 & $3.0-2.0$ & $0.73 \pm 0.22^{\text {abcdefg }}$ & 53.30 \\
\hline $0.5-3.0$ & $0.67 \pm 0.46^{\text {bcdefgh }}$ & 53.30 & $0.5-3.0$ & $0.40 \pm 0.22^{\text {efght }}$ & 40.00 & $0.5-3.0$ & $0.53 \pm 0.11^{\text {cdefghı }}$ & 53.30 \\
\hline $1.0-3.0$ & $0.80 \pm 0.34^{\text {abcdef }}$ & 66.60 & $1.0-3.0$ & $0.47 \pm 0.23^{\text {defghı }}$ & 40.00 & $1.0-3.0$ & $0.20 \pm 0.11^{\mathrm{h} 1}$ & 20.00 \\
\hline $2.0-3.0$ & $0.73 \pm 0.41^{\text {abcdefg }}$ & 53.30 & $2.0-3.0$ & $0.53 \pm 0.11^{\text {cdefght }}$ & 40.00 & $2.0-3.0$ & $0.40 \pm 0.20^{\text {efght }}$ & 46.60 \\
\hline $3.0-3.0$ & $0.80 \pm 0.20^{\mathrm{abcdef}}$ & 53.30 & $3.0-3.0$ & $0.73 \pm 0.30^{\text {abcdefg }}$ & 46.60 & $3.0-3.0$ & $0.40 \pm 0.20^{\mathrm{efgh} 1}$ & 40.00 \\
\hline
\end{tabular}


Table 1 (Continue)- Shoot regeneration from coleoptile explants cultured on MS medium containing different combinations of KIN, TDZ or BA with NAA, 2.4 D or IAA. Mean-values with the same letters within vertical columns are not significantly different (P>0.05). Control means are with no PGR treatment

\begin{tabular}{|c|c|c|c|c|c|c|c|c|}
\hline $\begin{array}{l}\text { PGRs } \\
\left(m g L^{-1)}\right.\end{array}$ & $\begin{array}{l}\text { Mean number } \\
\text { of shoots/explant }\end{array}$ & $\begin{array}{l}\text { Shoot } \\
\text { formation } \\
\text { percentage (\%) }\end{array}$ & $\begin{array}{l}\text { PGRs } \\
\left(m g L^{-1)}\right.\end{array}$ & $\begin{array}{l}\text { Mean number of } \\
\text { shoots/explant }\end{array}$ & $\begin{array}{l}\text { Shoot } \\
\text { formation } f \\
\text { percentage }(\%)\end{array}$ & $\begin{array}{l}\text { PGRs } \\
\left(m g L^{-1)}\right.\end{array}$ & $\begin{array}{l}\text { Mean number of } \\
\text { shoots/explant }\end{array}$ & $\begin{array}{l}\text { Shoot } \\
\text { formation } \\
\text { percentage (\%) }\end{array}$ \\
\hline Control & - & - & & - & - & & - & - \\
\hline $\begin{array}{l}T D Z- \\
2,4-D\end{array}$ & & & $\begin{array}{l}K I N-2,4 \\
D\end{array}$ & & & $\begin{array}{l}B A- \\
2,4-D\end{array}$ & & \\
\hline $0.5-0.5$ & $0.80 \pm 0.20^{\text {abcdef }}$ & 73.30 & $0.5-0.5$ & $0.67 \pm 0.31^{\text {bcdefgh }}$ & 53.30 & $0.5-0.5$ & $0.33 \pm 0.12^{\text {fght }}$ & 33.30 \\
\hline $1.0-0.5$ & $0.53 \pm 0.23^{\text {cdefgh } 1}$ & 46.60 & $1.0-0.5$ & $0.60 \pm 0.20^{\text {bcdefgh } 1}$ & 60.00 & $1.0-0.5$ & $0.67 \pm 0.61^{\text {bcdefgh }}$ & 53.30 \\
\hline $2.0-0.5$ & $0.67 \pm 0.31^{\text {bcdefgh }}$ & 60.00 & $2.0-0.5$ & $0.60 \pm 0.20^{\text {bcdefgh } 1}$ & 60.00 & $2.0-0.5$ & $0.47 \pm 0.42^{\text {defgh } 1}$ & 46.60 \\
\hline $3.0-0.5$ & $0.60 \pm 0.20^{\text {bcdefgh } 1}$ & 60.00 & $3.0-0.5$ & $0.53 \pm 0.31^{\text {cdefght }}$ & 53.30 & $3.0-0.5$ & $0.40 \pm 0.20^{\text {efght }}$ & 40.00 \\
\hline $0.5-1.0$ & $0.67 \pm 0.42^{\text {bcdefgh }}$ & 60.00 & $0.5-1.0$ & $0.73 \pm 0.12^{\text {abcdefg }}$ & 73.30 & $0.5-1.0$ & $0.40 \pm 0.20^{\text {efght }}$ & 40.00 \\
\hline $1.0-1.0$ & $0.87 \pm 0.12^{\text {abcde }}$ & 86.60 & $1.0-1.0$ & $0.67 \pm 0.12^{\text {bcdefgh }}$ & 66.60 & $1.0-1.0$ & $0.67 \pm 0.31^{\text {bcdefgh }}$ & 60.00 \\
\hline $2.0-1.0$ & $0.93 \pm 0.12^{\mathrm{abcd}}$ & 86.60 & $2.0-1.0$ & $0.53 \pm 0.12^{\text {cdefght }}$ & 53.30 & $2.0-1.0$ & $0.47 \pm 0.12^{\text {defgh } 1}$ & 40.00 \\
\hline $3.0-1.0$ & $0.73 \pm 0.23^{\text {abcdefg }}$ & 66.60 & $3.0-1.0$ & $0.60 \pm 0.40^{\text {bcdefght }}$ & 53.30 & $3.0-1.0$ & $0.33 \pm 0.23^{\text {fght }}$ & 33.30 \\
\hline $0.5-2.0$ & $0.73 \pm 0.12^{\text {abcdefg }}$ & 66.60 & $0.5-2.0$ & $0.40 \pm 0.40^{\text {efgh1 }}$ & 40.00 & $0.5-2.0$ & $0.20 \pm 0.00^{\mathrm{hl}}$ & 20.00 \\
\hline $1.0-2.0$ & $0.67 \pm 0.23^{\text {bcdefgh }}$ & 66.60 & $1.0-2.0$ & $0.33 \pm 0.31^{\text {fght }}$ & 33.30 & $1.0-2.0$ & $0.60 \pm 0.20^{\text {bcdefgh } 1}$ & 60.00 \\
\hline $2.0-2.0$ & $0.67 \pm 0.31^{\text {bcdefgh }}$ & 66.60 & $2.0-2.0$ & $0.60 \pm 0.00^{\text {bcdefgh }}$ & 60.00 & $2.0-2.0$ & $0.53 \pm 0.12^{\text {cdefgh } 1}$ & 53.30 \\
\hline $3.0-2.0$ & $0.53 \pm 0.12^{\text {cdefghı }}$ & 53.30 & $3.0-2.0$ & $0.47 \pm 0.31^{\text {defght }}$ & 46.60 & $3.0-2.0$ & $0.93 \pm 0.12^{\mathrm{abcd}}$ & 80.00 \\
\hline $0.5-3.0$ & $0.67 \pm 0.23^{\text {bcdefgh }}$ & 66.60 & $0.5-3.0$ & $0.60 \pm 0.20^{\text {bcdefgh } 1}$ & 60.00 & $0.5-3.0$ & $0.60 \pm 0.00^{\text {bcdefgh } 1}$ & 60.00 \\
\hline $1.0-3.0$ & $0.73 \pm 0.12^{\text {abcdefg }}$ & 73.30 & $1.0-3.0$ & $0.33 \pm 0.12^{\text {fght }}$ & 33.30 & $1.0-3.0$ & $0.27 \pm 0.31^{\text {ght }}$ & 20.00 \\
\hline $2.0-3.0$ & $0.47 \pm 0.12^{\text {defgh } 1}$ & 46.60 & $2.0-3.0$ & $0.40 \pm 0.00^{\text {efght }}$ & 40.00 & $2.0-3.0$ & $0.67 \pm 0.12^{\text {bcdefgh }}$ & 53.30 \\
\hline $3.0-3.0$ & $0.13 \pm 0.12^{1}$ & 20.00 & $3.0-3.0$ & $0.47 \pm 0.12^{\text {defgh } 1}$ & 46.60 & $3.0-3.0$ & $0.60 \pm 0.00^{\text {bcdefgh } 1}$ & 60.00 \\
\hline $\begin{array}{l}\text { TDZ - } \\
\text { NAA }\end{array}$ & & & $\begin{array}{l}\text { KIN } \\
\text { NAA }\end{array}$ & & & $\begin{array}{l}\text { BA - } \\
\text { NAA }\end{array}$ & & \\
\hline $0.5-0.5$ & $0.33 \pm 0.12^{\text {fght }}$ & 33.30 & $0.5-0.5$ & $0.67 \pm 0.12^{\text {bcdefgh }}$ & 66.60 & $0.5-0.5$ & $0.67 \pm 0.12^{\text {bcdefgh }}$ & 66.60 \\
\hline $1.0-0.5$ & $1.00 \pm 0.20^{\mathrm{abc}}$ & 66.60 & $1.0-0.5$ & $0.73 \pm 0.31^{\text {abcdefg }}$ & 73.30 & $1.0-0.5$ & $0.87 \pm 0.23^{\text {abcde }}$ & 73.30 \\
\hline $2.0-0.5$ & $0.73 \pm 0.12^{\text {abcdefg }}$ & 66.60 & $2.0-0.5$ & $0.93 \pm 0.12^{\mathrm{abcd}}$ & 93.30 & $2.0-0.5$ & $0.67 \pm 0.12^{\text {bcdefgh }}$ & 66.60 \\
\hline $3.0-0.5$ & $0.47 \pm 0.12^{\text {defght }}$ & 46.60 & $3.0-0.5$ & $0.27 \pm 0.46^{\text {ght }}$ & 26.60 & $3.0-0.5$ & $0.80 \pm 0.20^{\text {abcdef }}$ & 66.60 \\
\hline $0.5-1.0$ & $1.07 \pm 0.12^{\mathrm{ab}}$ & 86.60 & $0.5-1.0$ & $0.47 \pm 0.12^{\text {defght }}$ & 46.60 & $0.5-1.0$ & $0.67 \pm 0.23^{\text {bcdefgh }}$ & 66.60 \\
\hline $1.0-1.0$ & $1.20 \pm 0.53^{a}$ & 80.00 & $1.0-1.0$ & $0.47 \pm 0.12^{\text {defght }}$ & 46.60 & $1.0-1.0$ & $0.60 \pm 0.20^{\text {cdefgh }}$ & 60.00 \\
\hline $2.0-1.0$ & $0.67 \pm 0.12^{\text {bcdefgh }}$ & 66.60 & $2.0-1.0$ & $0.53 \pm 0.31^{\text {cdefght }}$ & 60.00 & $2.0-1.0$ & $0.87 \pm 0.12^{\text {abcde }}$ & 73.30 \\
\hline $3.0-1.0$ & $0.67 \pm 0.12^{\text {bcdefgh }}$ & 66.60 & $3.0-1.0$ & $0.80 \pm 0.20^{\text {abcdef }}$ & 60.00 & $3.0-1.0$ & $0.33 \pm 0.31^{\text {fght }}$ & 33.30 \\
\hline $0.5-2.0$ & $0.67 \pm 0.12^{\text {bcdefgh }}$ & 80.00 & $0.5-2.0$ & $0.33 \pm 0.12^{\text {fght }}$ & 26.60 & $0.5-2.0$ & $1.00 \pm 0.53^{\mathrm{abc}}$ & 66.60 \\
\hline $1.0-2.0$ & $0.80 \pm 0.40^{\text {abcdef }}$ & 60.00 & $1.0-2.0$ & $0.67 \pm 0.31^{\text {bcdefgh }}$ & 60.00 & $1.0-2.0$ & $0.53 \pm 0.12^{\text {cdefght }}$ & 53.30 \\
\hline $2.0-2.0$ & $0.67 \pm 0.12^{\text {bcdefgh }}$ & 60.00 & $2.0-2.0$ & $0.53 \pm 0.12^{\text {cdefght }}$ & 53.30 & $2.0-2.0$ & $0.60 \pm 0.20^{\text {cdefghı }}$ & 60.00 \\
\hline $3.0-2.0$ & $0.87 \pm 0.12^{\text {abcde }}$ & 80.00 & $3.0-2.0$ & $0.27 \pm 0.23^{\mathrm{ght}}$ & 26.60 & $3.0-2.0$ & $0.93 \pm 0.12^{\mathrm{abcd}}$ & 73.30 \\
\hline $0.5-3.0$ & $0.73 \pm 0.12^{\text {abcdefg }}$ & 73.30 & $0.5-3.0$ & $0.60 \pm 0.20^{\text {bcdefgh } 1}$ & 53.30 & $0.5-3.0$ & $0.53 \pm 0.31^{\text {cdefght }}$ & 53.30 \\
\hline $1.0-3.0$ & $0.67 \pm 0.12^{\text {bcdefgh }}$ & 66.60 & $1.0-3.0$ & $0.53 \pm 0.12^{\text {cdefght }}$ & 53.30 & $1.0-3.0$ & $0.67 \pm 0.12^{\text {bcdefgh }}$ & 66.60 \\
\hline $2.0-3.0$ & $0.73 \pm 0.12^{\text {abcdefg }}$ & 73.30 & $2.0-3.0$ & $0.47 \pm 0.31^{\text {defght }}$ & 46.60 & $2.0-3.0$ & $0.53 \pm 0.12^{\text {cdefghı }}$ & 66.60 \\
\hline $3.0-3.0$ & $0.67 \pm 0.12^{\text {bcdefgh }}$ & 66.60 & $3.0-3.0$ & $0.27 \pm 0.12^{\mathrm{ghr}}$ & 26.60 & $3.0-3.0$ & $0.80 \pm 0.00^{\text {abcdef }}$ & 80.00 \\
\hline
\end{tabular}

Recent research found that immature and mature zygotic embryos of einkorn could induce embryogenic callus to a satisfactory level, however, the number of regenerated shoots was less than 0.5 per explant (Özgen et al. 2017) or there was no information regarding the number of regenerated shoots (Yang et al. 2015). Örgeç et al. (2021) found that coleoptile explants 
showed the greatest performance for callus induction and indirect plant regeneration compared with root and leaf explants. Furthermore, it was also reported that callus cultures derived from coleoptile explants were capable of plant regeneration whereas callus cultures derived from leaf and root explants were not. A similar result by Sarker \& Biswas (2002), stating that root explants of Triticum aestivum L. wheat cultivars developed callus that could not regenerate plants, whereas the leaf explants did not produce callus. Benkirane et al. (2000) also showed that the coleoptile explants of Triticum turgidum ssp. durum. had a higher callus percentage and plant regeneration potential. All these investigations show that the type of explant has a major impact on the regeneration capability. Our findings corroborate previous findings that coleoptile explants were suitable for shoot regeneration and outperformed all other explant types evaluated.

The supporting effect of TDZ on the plant regeneration process has been recently reported for many species (Phippen \& Simon 2000; Yucesan et al. 2007; Wang \& Bao 2007; Ekmekci \& Aasim 2014). Improved regeneration protocols using varied concentrations of thidiazuron (TDZ) applied alone or in conjunction with other plant growth regulators were also developed for a variety of polyploid wheat cultivars (Shan et al. 2000; Ganeshan et al. 2006; She et al. 2013). Miroshnichenko et al. (2016) reported that TDZ enhanced the regeneration capacity of embriyonic callus in cultures of T. kiharae. Benlioğlu \& Birsin (2017) reported that TDZ had a positive effect on plant regeneration from immature embryo-derived T. aestivum L. callus. Different concentrations of TDZ promoted plant regeneration from einkorn callus derived from immature embryo explants (Miroshnichenko et al. 2017). Callus derived from coleoptile explants of T. monococcum L. was stimulated by TDZ to regenerate plants (Örgeç et al. 2021). Similarly, in our study, TDZ was a more influential hormone than BA or KIN on the shoot regeneration process (Table 1).

In the second stage of our study, shoots were subcultured on media containing all effective hormonal combinations for 45 days (Table 2). It was noteworthy that 3.66 shoots per explant were induced by the MS medium containing $1 \mathrm{mg} \mathrm{L}^{-1} \mathrm{TDZ}$ plus 1 $\mathrm{mg} \mathrm{L}^{-1} \mathrm{NAA}$ and $2 \mathrm{mg} \mathrm{L}^{-1} \mathrm{KIN}$ plus $0.5 \mathrm{mg} \mathrm{L}^{-1} \mathrm{NAA}$ for 45 days (Figure 2). Induction media comprising $2 \mathrm{mg} \mathrm{L}^{-1} 2.4-\mathrm{D}$ plus 3 $\mathrm{mg} \mathrm{L}^{-1} \mathrm{BA}$ was found inappropriate for continuous shoot formation. However, the callus formation was observed. This observation is in line with several studies (Dale \& Deambrogio 1979; Benkirane et al. 2000; Sarker \& Biswas 2002; Alikina et al. 2016). It was found that an increased concentration of $2.4 \mathrm{D}$ induced callusing in Triticum aestivum L. (Mahmood et al. 2012) and T. monococcum L. (Örgeç et al. 2021). 2,4-D is an auxin-like plant growth regulator that is commonly used in cereals for callus production however it displays low effect for improving somatic embryogenesis and plant regeneration (Miroshnichenko et al. 2017). Similarly, in our study, interaction with 2.4 D increased the callusing potential while decreasing shoot formation capacity. The variability in callus formation frequency in return for various levels of 2.4-D may be due to differences in genes controlling callusing or genes may not express themselves fully in some cultivars contrary to others supplemented with an optimum concentration of 2.4-D. Our findings are in concurrence with other researchers who also suggested genotypic differences of wheat for callus formation and regeneration abilities (Kilinc 2004; Nasircilar et al. 2006; Hassan et al. 2009; Örgeç et al. 2021). Although various researchers had standardized the concentration of 2.4-D at the optimum level for diverse genotypes of wheat (Satyavathi et al. 2004; Sarker \& Biswas 2002) the induction media should be standardized for maximum callusing in einkorn wheat.

Table 2- Shoot regeneration obtained from in vitro-grown regenerants subcultured on MS medium containing the most effective hormone combination. Mean values $( \pm$ SD) with the different letters in the same columns are significantly different $(P<0.05)$

\begin{tabular}{|c|c|c|}
\hline $\begin{array}{l}\text { Combinations of } \\
\text { PGRs }\end{array}$ & $\begin{array}{l}\text { Mean number of } \\
\text { shoots/explant }\end{array}$ & $\begin{array}{l}\text { Shoot formation percentage } \\
(\%)\end{array}$ \\
\hline Control & $0.66^{\mathrm{ab}} \pm 0.20$ & 26.66 \\
\hline $0.5 \mathrm{mg} \mathrm{L}^{-1} \mathrm{TDZ}$ & $2.33^{\mathrm{a}} \pm 0.88$ & 73.33 \\
\hline $1 \mathrm{mg} \mathrm{L}^{-1} \mathrm{TDZ}+1 \mathrm{mg} \mathrm{L}^{-1} \mathrm{NAA}$ & $3.66^{a} \pm 0.66$ & 73.33 \\
\hline $2 \mathrm{mg} \mathrm{L}^{-1} \mathrm{TDZ}$ & $3.00^{\mathrm{a}} \pm 0.33$ & 46.66 \\
\hline $0.5 \mathrm{mg} \mathrm{L}^{-1} \mathrm{BA}+2 \mathrm{mg} \mathrm{L}^{-1} \mathrm{NAA}$ & $2.66^{\mathrm{a}} \pm 0.57$ & 60.00 \\
\hline $3 \mathrm{mg} \mathrm{L}^{-1} \mathrm{BA}+0.5 \mathrm{mg} \mathrm{L}^{-1} \mathrm{IAA}$ & $3.33^{\mathrm{a}} \pm 0.88$ & 66.60 \\
\hline $3 \mathrm{mg} \mathrm{L}^{-1} \mathrm{BA}+2 \mathrm{mg} \mathrm{L}^{-1} 2.4-\mathrm{D}$ & $0.46^{\mathrm{ab}} \pm 0.33$ & 60.00 \\
\hline $0.5 \mathrm{mg} \mathrm{L}^{-1} \mathrm{KIN}+1 \mathrm{mg} \mathrm{L}^{-1} 2.4-\mathrm{D}$ & $3.00^{\mathrm{a}} \pm 0.57$ & 33.33 \\
\hline $1 \mathrm{mg} \mathrm{L}^{-1} \mathrm{KIN}$ & $3.00^{\mathrm{a}} \pm 1.00$ & 60.00 \\
\hline $2 \mathrm{mg} \mathrm{L}^{-1} \mathrm{KIN}+0.5 \mathrm{mg} \mathrm{L}^{-1} \mathrm{NAA}$ & $3.66^{\mathrm{a}} \pm 0.33$ & 60.00 \\
\hline
\end{tabular}




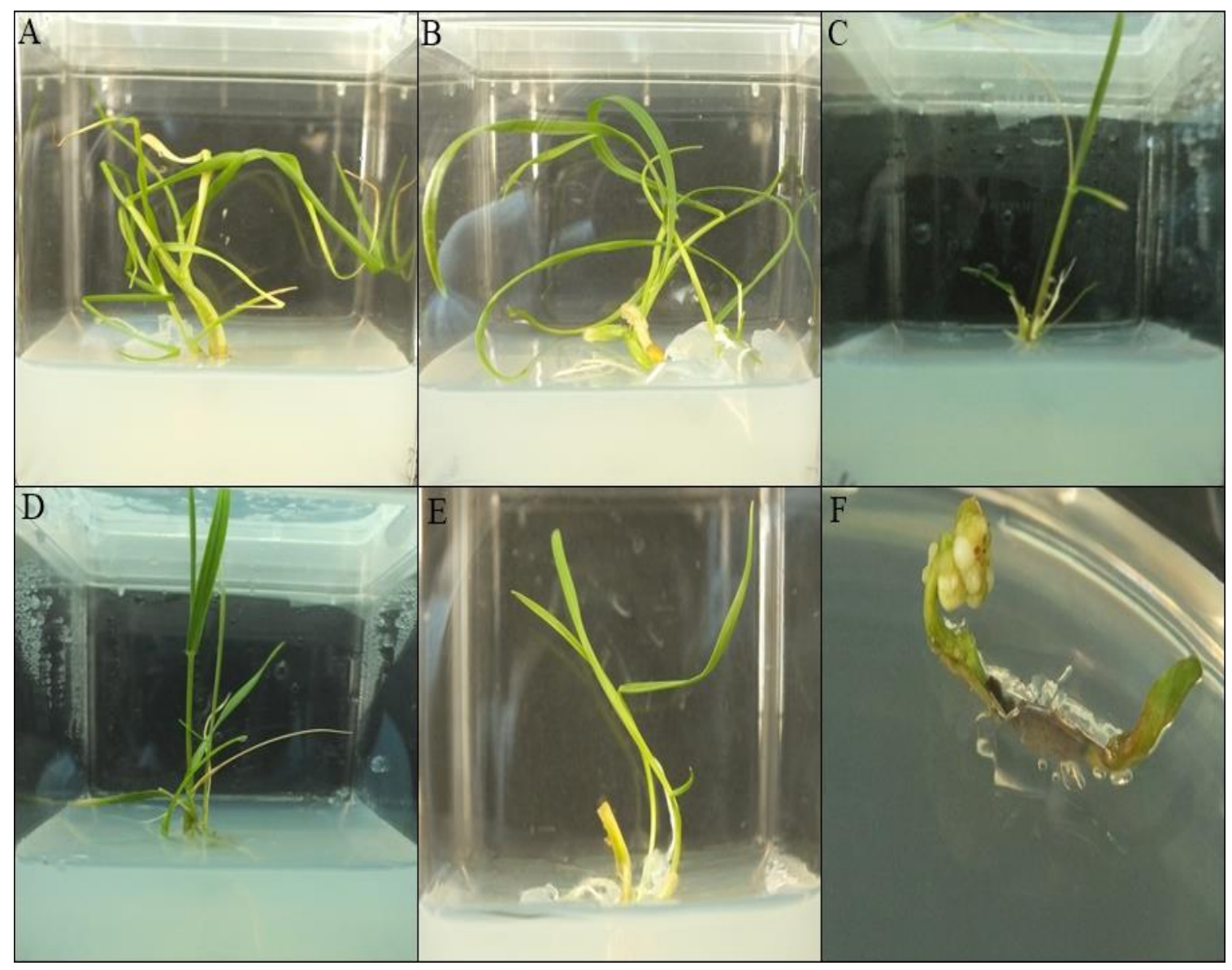

Figure 2- The shoots were subcultured on the MS medium containing the most effective hormone combinations. (A) 2.0 mg $\mathrm{L}^{-1}$ KIN + 0.5 mg L ${ }^{-1} \mathrm{NAA}$ (B) $1.0 \mathrm{mg} \mathrm{L}^{-1} \mathrm{TDZ}+1.0 \mathrm{mg} \mathrm{L}^{-1} \mathrm{NAA}$. (C) $0.5 \mathrm{mg} \mathrm{L}^{-1} \mathrm{TDZ}$. (D) $0.5 \mathrm{mg} \mathrm{L}^{-1} \mathrm{TDZ}^{+} \mathbf{2 . 0} \mathrm{mg} \mathrm{L}^{-1} \mathrm{NAA}$. (E) 1.0 mg L $\mathrm{L}^{-1} \mathrm{KIN}$. (F) $3.0 \mathrm{mg} \mathrm{L}^{-1} \mathrm{BA}+2.0 \mathrm{mg} \mathrm{L}^{-1} 2.4-\mathrm{D}$

Early in the 1930s, indole-3-acetic acid (IAA) was identified to be the most effective auxin in promoting the development of adventitious root (Thimann \& Koepfli 1935), and since then IAA has been widely used to induce adventitious root formation in the clonal propagation of various species (Shu et al. 2019). Although only a few studies have focused on the root formation for in vitro study of wheat, IAA has been one of the most widely used auxin-hormone to induce root induction (Kopertekh \& Stribnaya 2003; Örgeç et al. 2021).

In our study, to induce root formation, regenerated shoots (45 days of culture) were cultured on MS medium with different concentrations of IAA ranging from 0.5 to $5 \mathrm{mg} \mathrm{L}^{-1}$ (Table 2). They formed roots in one week. Among the different IAA concentrations tested, $2 \mathrm{mg} \mathrm{L}^{-1}$ IAA was the most effective hormone concentration for rooting (Figure 3). 12.33 \pm 0.88 roots produced per regenerated shoot. When IAA concentration was increased from 0.5 to $2 \mathrm{mg} \mathrm{L}^{-1}$, more root formation was observed. However, when IAA concentration was increased from 2 to $5 \mathrm{mg} \mathrm{L}^{-1}$, the mean number of roots began to decline (Table 3). To get the acquisition of the meristematic competence of the cells, auxin is accepted as the most effective hormone in tissue culture experiments. However, it was known that after this competence was established, excessive auxin concentration inhibited further adventitious or embryonic root development (Gurel \& Wren 1995; Charriere et al. 1999).

Table 3- Effects of the tested auxins on rooting. Mean values $( \pm$ SD) with the different letters in the same columns are significantly different $(\mathbf{P}<\mathbf{0 . 0 5})$

\begin{tabular}{|c|c|c|}
\hline Auxin concentration & Mean number of roots/shoot & Root formation percentage (\%) \\
\hline $0.5 \mathrm{mg} \mathrm{L}^{-1}$ IAA & $9.00^{\mathrm{b}} \pm 1.00$ & 100 \\
\hline $1 \mathrm{mg} \mathrm{L}^{-1} \mathrm{IAA}$ & $11.67^{\mathrm{a}} \pm 0.66$ & 100 \\
\hline $2 \mathrm{mg} \mathrm{L}^{-1}$ IAA & $12.33^{\mathrm{a}} \pm 0.88$ & 100 \\
\hline $3 \mathrm{mg} \mathrm{L}^{-1}$ IAA & $7.33^{\mathrm{bc}} \pm 0.33$ & 100 \\
\hline $5 \mathrm{mg} \mathrm{L}^{-1}$ IAA & $5.33^{c} \pm 0.88$ & 100 \\
\hline
\end{tabular}






Figure 3- Effect of $2 \mathrm{mg} \mathrm{L}^{-1}$ IAA on root formation from regenerated shoots

Finally, these well-developed plantlets were transferred to plastic pots containing a mixture of vermiculite and soil (1:2). For acclimatization, they were kept in the climate room for one week. After one week, the plantlets were transferred to pots containing commercial soil, kept under room conditions (Figure 4. a-f). Eventually, all the plantlets were established in the field, with 100\% survival. 


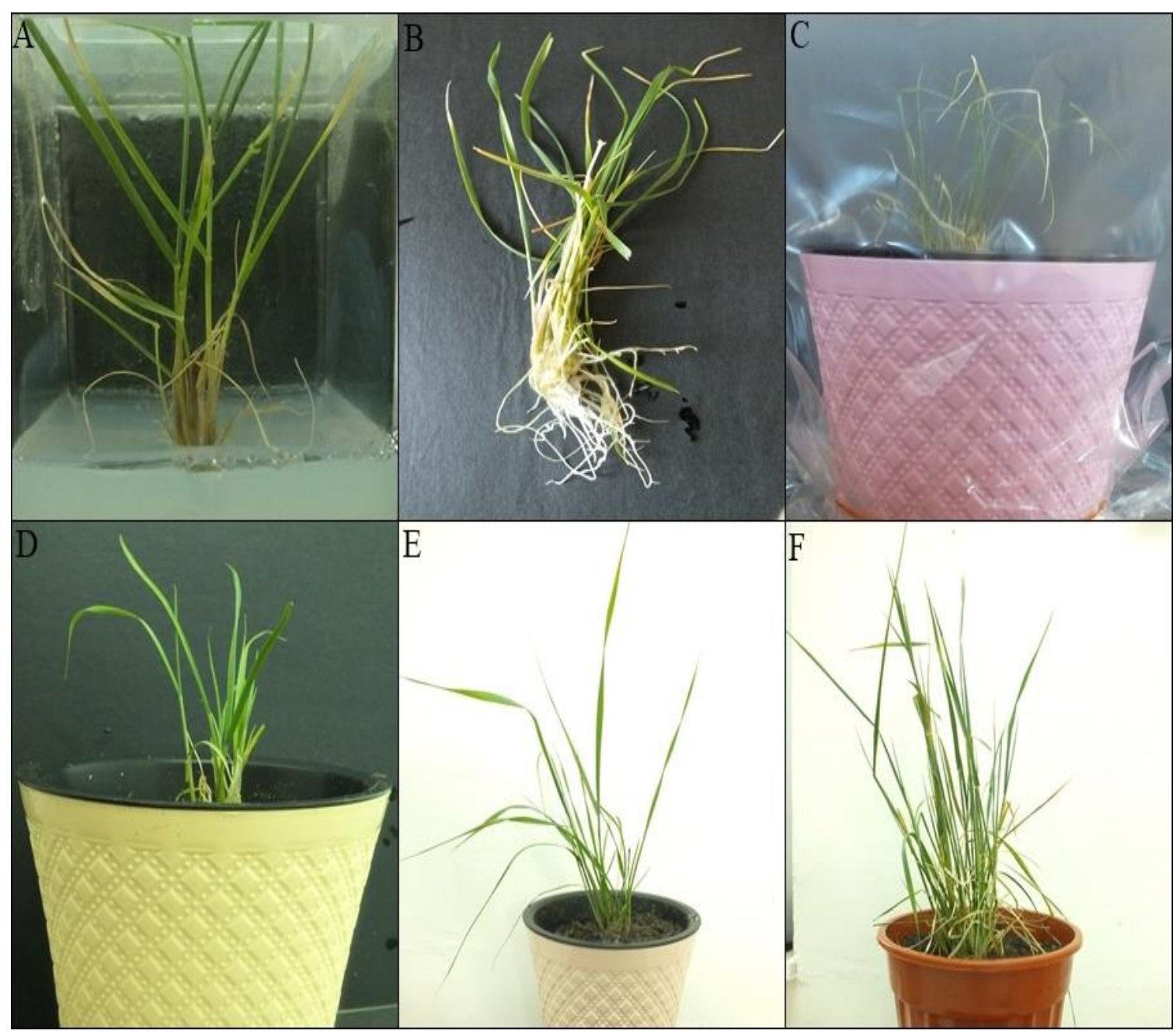

Figure 4- Direct shoot and plant regeneration from coleoptile explants of Triticum monococcum L. (A) Rooting of the regenerated shoots on medium containing $2.0 \mathrm{mg} \mathrm{l}^{-1}$ IAA after three weeks in culture. (B-C) Regenerated plants transferred to pots containing a mixture of vermiculite and soil (1:2) under climate room conditions. (D-F) Regenerated plants transferred to pots containing sterile soil under room conditions.

\section{Conclusions}

This article describes an efficient protocol for direct plant regeneration using somatic tissues in einkorn by testing different concentrations of TDZ, KIN, and BAP in combination with NAA, IAA, and 2.4-D. The present investigation elucidates that the coleoptile part of wheat can serve as a potential source for direct shoot regeneration under suitable conditions. This method is an efficient one for the ex vitro conservation of kinds of ancient wheat genetic resources and clonal propagation.

\section{Acknowledgments}

This study was supported by the Bolu Abant İzzet Baysal University Research Foundation (Project No: BAP - 2017.03.01.1130). The authors thank to the Bolu Municipality (2018/2019) for a research grant.

\section{References}

Alikina O, Chernobrovkina M, Dolgov S \& Miroshnichenko D (2016). Tissue culture efficiency of wheat species with different genomic formulas. Crop Breeding and Applied Biotechnology 16: 307-314. https://doi.org/10.1590/1984-70332016v16n4a46

Benkirane H, Sabounji K, Chlyah A \& Chlyah H (2000). Somatic embryogenesis and plant regeneration from fragments of immature inflorescences and coleoptiles of durum wheat. Plant Cell Tissue Organ Culture 61: 107-113. https://doi.org/10.1023/a:1006464208686

Benlioğlu B \& Birsin M A (2017). A thidizuron (TDZ) - based efficient plant regeneration system from callus cultures, obtained through various embryo sources, in common winter wheat (Triticum aestivum L.). Ciência e Técnica Vitivinícola 32: 108-119

Bi R \& Wang H (2008). Primary studies on tissue culture of mature embryo from diploid and tetraploid wheat. Frontiers of Agriculture in China 2: 262-265. https://doi.org/10.1007/s11703-008-0038-y

Bidabadi S S \& Jain S M (2020). Cellular, molecular, and physiological aspects of in vitro plant regeneration. Plants 9:702-722. https://doi.org/10.3390/plants9060702 
Charriere F, Sotta B, Emile M \& Günther H (1999). Induction of adventitious shoots or somatic embryos on in vitro cultured zygotic embryos of Helianthus annuus: variation of endogenous hormone levels. Plant Physiology Biochemistry 37: 751-757. https://doi.org/10.1016/s0981-9428(00)86688-7

Chauhan H, Desai S A \& Khurana P (2007). Comparative analysis of the differential regeneration response of various genotypes of Triticum aestivum, Triticum durum and Triticum dicoccum. Plant Cell Tissue and Organ Culture 91: 191-199. https://doi.org/10.1007/s11240-0079285-5

Dale P J \& Deambrogio E (1979). A Comparison of callus induction and plant regeneration from different explants of Hordeum vulgare. Zeitschrift für Pflanzenphysiologie 94: 65-77. https://doi.org/10.1016/s0044-328x(79)80249-4

Ekmekci H \& Aasim M (2014). In vitro plant regeneration of Turkish sweet basil (Ocimum basilicum). The Journal of Animal \& Plant Sciences 24(6): 1758-1765

Fennell S, Bohorova N, Ginkel M V, Crossa J \& Hoisington D (1996). Plant regeneration from immature embryos of 48 elite CIMMYT bread wheats. Theoretical and Applied Genetics 92: 163-169. https://doi.org/10.1007/bf00223371

Goncharov N P (2011). Genus Triticum L. taxonomy: the present and the future. Plant Systematics and Evolution 295: 1-11

Gurel E \& Wren M J (1995). In vitro development from leaf explants of sugar beet (Beta vulgaris L.) Rhizogenesis and the effect of sequential exposure to auxin and cytokinin. Annual Botany 75:31-38. https://doi.org/10.1016/s0305-7364(05)80006-x

Hassan M U, Ahmed Z, Munir M, Malik S I \& Shahzad K (2009). Effect of sorbitol in callus induction and plant regeneration in wheat. African Journal of Biotechnology 8(23): 6529-6535

Hidalgoa A, Andrea B A, Pompeia C \& Piscozzia R (2006). Carotenoids and tocols of einkorn wheat (Triticum monococcum ssp. monococcum L.). Journal of Cereal Science 44: 182-193. https://doi.org/10.1016/j.jcs.2006.06.002

Kilinc M (2004). Effects of dicamba concentration on the embryo cultures of some bread wheat (Triticum aestivum L.) genotypes. Biotechnology \& Biotechnology Equipment 58-61. https://doi.org/10.1080/13102818.2004.10817087

Kopertekh L G \& Stribnaya A (2003). Regeneration from wheat leaf explants. Russian Journal of Plant Physiology 50(3): 365-369

Lazar M D, Collins G B \& Vian W E (1983). Genetic and environmental effects on the growth and differentiation of wheat somatic cell cultures. Journal of Heredity 74: 353-357. https://doi.org/10.1093/oxfordjournals.jhered.a109809

Mahmood I A, Razzaq Z, Khan I, Hafiz A \& Kaleem S (2012). Evaluation of tissue culture responses of promising wheat (Triticum aestivum L.) cultivars and development of an efficient regeneration system. Pakistan Journal of Botany 44: 277-284

Miroshnichenko D, Chernobrovkina M \& Dolgov S (2016). Somatic embryogenesis and plant regeneration from immature embryos of Triticum timopheevii and Triticum kiharae Dorof. et Migusch, wheat species with G genome. Plant Cell Tissue and Organ Culture 125: 495-508. https://doi.org/10.1007/s11240-016-0965-x

Miroshnichenko D, Chaban I, Chernobrovkina M \& Dolgov S (2017). Protocol for efficient regulation of in vitro morphogenesis in einkorn (Triticum monococcum L.), a recalcitrant diploid wheat species. Plos One 12(3): e0173533 https://doi.org/10.1371/journal.pone.0173533

Murashige T \& Skoog F (1962). A revised medium for rapid growth and bioassays with tobacco tissue cultures. Physiology and Plantarum 15: 473-497. https://doi.org/10.1111/j.1399-3054.1962.tb08052.x

Nasircilar A G, Turgu K \& Fiskin K (2006). Callus induction and plant regeneration from mature embryos of different wheat genotypes. Pakistan Journal of Botany 2006; 38(2): 637-645

Nesbitt M \& Samuel D (1996). From staple crop to extinction? The archaeology and history of the hulled wheat. In: Padulosi S, Hammer K, Heller J (eds) Hulled wheats, promoting the conservation and used of underutilized and neglected crops. IPGRI, Rome 1996; p. 40-99

Nevo E (2011). Triticum. In Kole C (ed) Wild crop relatives: genomic and breeding resources, cereals. Springer Verlag, Berlin, 2011; p. 407456. https://doi.org/10.1007/978-3-642-14228-4_10

Örgeç M, Karakaş F P, Şahin G, Ağıl F \& Zencirci N (2018). Einkorn (Triticum monococcum spp. monococcum) In Vitro propagation sterilization protocol. International Journal of Secondary Metabolite 5(2): 67-74. https://doi.org/10.21448/ijsm.399094

Örgeç M, Verma S K, Şahin G, Zencirci N \& Gürel E (2021) In vitro tissue culture protocol of ancient einkorn (Triticum monococcum ssp. monococum) wheat via indirect shoot regeneration. In Vitro Cellular \& Developmental Biology - Plant 57: 143-151. https://doi.org/10.1007/s11627-020-10122-8

Özgen M, Birsin M A \& Benlioglu B (2017). Biotechnological characterization of a diverse set of wheat progenitors (Aegilops sp. and Triticum sp.) using callus culture parameters. Plant Genetic Resources 15(01):45-50. https://doi.org/10.1017/s1479262115000350

Phippen W B \& Simon J E (2000). Shoot regeneration of young leaf explants from basil (Ocimum basilicum L.). In Vitro Cellular \& Developmental Biology - Plant 36: 250-254. https://doi.org/10.1007/s11627-000-0046-y

Sarker R H \& Biswas A (2002). In vitro plantlet regeneration and Agrobacterium-mediated genetic transformation of wheat (Triticum aestivum L.). Plant Tissue Culture 12: 155- 165.

Satyavathi V V, Jauhar P P, Elias E M \& Rao M B (2004). Effects of growth regulators on in vitro plant regeneration in durum wheat. Crop Science 44: 1839-1846. https://doi.org/10.2135/cropsci2004.1839

Shan X Y, Li D S \& Qu R D (2000). Thidiazuron promotes in vitro regeneration of wheat and barley. In Vitro Cellular \& Development Biology - Plant 36: 207-210. https://doi.org/10.1007/s11627-000-0038-y

She M Y, Yin G X, Li J R, Li X, Du L P \& Ma W J (2013). Efficient regeneration potential is closely related to auxin exposure time and catalase metabolism during the somatic embryogenesis of immature embryos in Triticum aestivum L. Molecular Biotechnology 54: 451460. https://doi.org/10.1007/s12033-012-9583-y

Shu W, Zhou H, Jiang C, Zhao S, Wang L, Li Q, Yang Z \& Groover A (2019). The auxin receptor TIR1 homolog (PagFBL1) regulates adventitious rooting through interactions with Aux/IAA28 in Populus. Plant Biotechnology Journal 17: 338-349. https://doi.org/10.1111/pbi.12980

Tama's C, Szucs P, Rakszegi M, Tama's L \& Bedö Z (2004). Effect of combined changes in culture medium and incubation conditions on the regeneration from immature embryos of elite varieties of winter wheat. Plant Cell Tissue and Organ Culture 79: 39-44. https://doi.org/10.1023/b:ticu.0000049447.81409.ed

Thimann K V \& Koepfli J B (1935). Identity of the growth promoting androot-forming substances of plants. Nature 135: 101-102. https://doi.org/10.1038/135101a0

Wang J \& Bao M (2007). Plant regeneration of pansy (Viola wittrockiana) 'Caidie' via petiole-derived callus. Scientia Horticulturae 111: 266270. https://doi.org/10.1016/j.scienta.2006.10.011

Yang S, Xu K, Wang Y, Bu B, Huang W, Sun F, Liu S \& Xi Y (2015). Analysis of biochemical and physiological changes in wheat tissue culture using different germplasms and explant types. Acta Physiologiae Plantarum 37: 1-10. https://doi.org/10.1007/s11738-015-1861-4 
Yucesan B, Turker A U \& Gurel E (2007). TDZ-induced high frequency plant regeneration through multiple shoot formation in witloof chicory (Cichorium intybus L.). Plant Cell Tissue and Organ Culture 91: 243-250. https://doi.org/10.1007/s11240-007-9290-8

(C) 2022 by the author(s). Published by Ankara University, Faculty of Agriculture, Ankara, Turkey. This is an Open Access article distributed under the terms and conditions of the Creative Commons Attribution (CC BY) license (http://creativecommons.org/licenses/by/4.0/), which permits unrestricted use, distribution, and reproduction in any medium, provided the original work is properly cited. 